\title{
Differential effects of EPA and DHA on plasma and adipose tissue lipid mediators: the ComparED study
}

\author{
$\underline{\text { Cécile Vors }}^{1}$, Fabiana Piscitelli ${ }^{2}$, Roberta Verde ${ }^{2}$, Sofia Laforest ${ }^{3,4}$, Janie Allaire ${ }^{1}$, \\ Johanne Marin ${ }^{1}$, Baptiste Fourmaux ${ }^{5}$, Nathalie Bernoud-Hubac ${ }^{5}$, Marie-Caroline Michalski ${ }^{5}$, \\ Patrick Couture $^{1,4}$, Dominique Fortin ${ }^{6}$, André Tchernof ${ }^{3,4}$, Vincenzo Di Marzo ${ }^{1,3}$ and \\ Benoît Lamarche ${ }^{1}$ \\ ${ }^{1}$ Institute of Nutrition and Functional Foods (INAF), Laval University, Quebec, Canada, \\ ${ }^{2}$ Institute of Biomolecular Chemistry, Consiglio Nazionale delle Ricerche, Naples, Italy, \\ ${ }^{3}$ University Institute of Cardiology and Pneumology, Quebec, Canada, \\ ${ }^{4} \mathrm{CHU}$ de Québec Research Center, Laval University, Quebec, Canada, \\ ${ }^{5}$ INRA UMR1397, INSERM U1060, INSA-Lyon, CarMeN laboratory, Villeurbanne, France and \\ ${ }^{6} \mathrm{CHU}$ de Québec St-François d'Assise Hospital, Quebec, Canada
}

\begin{abstract}
Oxylipins and endocannabinoids (eCBs) both belong to superclasses of lipid mediators with potent inflammation modulatory activities. The adipose tissue (AT) plays a key role in metabolic syndrome-related inflammation, via altered adipocyte physiology, infiltrated macrophages and altered profile of eCBs. We previously reported that DHA is more potent than EPA at modulating systemic inflammation, but the underlying mechanisms remain unclear. The objective of this study was to compare the individual effect of high-dose DHA and of EPA on circulating lipid mediators, i.e. plasma oxylipins and eCBs, and AT-related inflammation. In a randomized double-blind crossover trial, 154 volunteers with abdominal obesity and low-grade inflammation were subjected to three 10-wk supplementation phases: 1- EPA (2.7 g/d); 2- DHA (2.7 g/d); 3- corn oil (control), each separated by a 9-wk washout. Supplements were provided as re-esterified triacylglycerols. Profiling of plasma oxylipins and eCBs was performed on 58 subjects after each phase. Abdominal subcutaneous AT biopsies were also obtained from 13 individuals after each phase. Plasma DHA-, EPA-, arachidonic acid-derived oxylipins were analyzed by LC-MS. eCBs and some of their bioactive congeners were analyzed in plasma and AT by LC-APCI-MS. Adipocyte diameter was determined by histological analysis and AT macrophage infiltration was quantified by double immunofluorescence. Compared with EPA, DHA increased plasma levels of hydroxy-docosahexaenoic acids (7-, 11-, 14-, 4-, 17-HDOHE; P < 0.0001) and palmitoylethanolamide (PEA; P = 0.04). Compared with DHA, EPA led to higher plasma level of hydroxy-eicosapentaenoic acids (12-, 15-, 5-HEPE; P<0.0001). In the AT, EPA increased the level of oleoylethanolamide $(\mathrm{OEA} ; \mathrm{P}=0.01)$ compared with $\mathrm{DHA}$, but no other difference was observed between treatments in adipose eCBs and eCB-related lipids. DHA and EPA did not differentially modify adipocyte size distributions $(\mathrm{P}>0.50)$ and proportions of M1-type and M2-type macrophages $(\mathrm{P}>0.30)$. In conclusion, increased plasma levels of anti-inflammatory DHA-derived oxylipins and plasma PEA may be responsible, at least to some extent, for the more potent anti-inflammatory effects of DHA compared with EPA observed in the ComparED study. Conversely, subcutaneous AT does not seem to be involved in explaining such differences between EPA and DHA.
\end{abstract}

\section{Conflict of Interest}

Douglas Laboratories provided the EPA, DHA and control capsules used in this study. 